Pak. j. sci. ind. res. Ser. A: phys. sci. 201255 (3) 135-141

\title{
Absorption Studies of Binary Mixtures
}

\author{
Sathi Reddy ${ }^{\mathrm{a}}$, Shanmukhi Jyothi ${ }^{\mathrm{b} *}$ and Linga Reddy ${ }^{\mathrm{c}}$ \\ ${ }^{\mathrm{a}}$ Eritrea Institute of Technology, Asmara, Eritrea, P.O. Box-12676, Africa \\ ${ }^{b}$ Department of Physics, Sardar Patel College, Secunderabad-500 025, (A.P), India \\ ${ }^{c}$ Department of Physics, UCS, Osmania University, Hyderabad -500 007, (A.P), India
}

(received July 2, 2011; revised October 28, 2011; accepted October 28, 2011)

\begin{abstract}
Ultrasonic velocity, density and viscosity for binary mixtures of Aniline with ethanol, methanol and propanol have been measured at room temperature of about $300.15 \mathrm{~K}$ over entire volume component percentage range. The ultrasonic velocity (v) is measured with ultrasonic Pulse Echo Overlap (PEO) technique at a frequency of $2 \mathrm{MHz}$. The density $(\rho)$ measurements have been carried out by $10 \mathrm{~mL}$ specific gravity bottle. The viscosity $(\eta)$ measurements have been carried out by using Cannon-Fenske Viscometer with an accuracy of $\pm 0.3 \%$. Attenuation coefficient $(\alpha)$ is measured using a cathode ray oscilloscope (CRO) for a transmitted pulse and $\mathrm{n}^{\text {th }}$ echo. Relaxation time $(\tau)$, absorptivity $(\mathrm{A})$ was computed using measured data of ultrasonic velocity, density and viscosity. Data of ultrasonic velocity, attenuation coefficient, density and viscosity are useful in evaluating some useful thermodynamic properties like adiabatic compressibility, internal pressure, free volume which can be utilized as a qualitative guide to predict the extent of complex formation in binary liquid mixtures of aniline-alcohol mixtures.
\end{abstract}

Keywords: ultrasonic velocity, density, viscosity, attenuation coefficient, absorptivity, relaxation time

\section{Introduction}

Considerable interest has been stimulated by the ultrasonic investigations of liquids and liquid mixtures. During the past four decades the measurement of the Velocity and attenuation of ultrasonic waves has been the basis of evolution of a wide variety of physical properties of gases, liquids and solids.

The ultrasonic measurements stand as one of the primary techniques for study of properties of matter such as mechanical, electromagnetic and particle interaction. The propagation behaviour of high frequency stress wave is determined by the measurement of velocity and attenuation of ultrasonic waves, as a function of environmental variables such as temperature, pressure etc. In recent years the measurements of ultrasonic studies have been adequately employed in understanding the nature of molecular interactions in pure liquids and liquid mixtures which are very important because of their extensive use in the textile industry and other ultrasonic studies on ternary liquid mixtures have been investigated by several workers (Durai and Ramadas, 2004; Tuulmets and Salmar, 2001; Anantakrishnan and Anantaraman, 1959; Amis, 1955; Nomoto, 1953).

*Author for correspondence; E-mail: jyothi6physics@gmail.com
The propagation of ultrasonic waves in a substance has become a fundamental test to investigate its properties. Ultrasonic velocity measurements have been adequately employed to understand the nature of molecular interactions in pure liquids, binary and the ionic interactions in aqueous electrolytic solutions. In a material, scattering and absorption is produced by various dynamical interactions which typically arise from enharmonic force between the atoms. The presence of free charge carriers, cycling to localized spin states and the presence of impurities show drastic changes in the binary mixtures which was reported by Chavez et al. (1986). When acoustic waves are propagated through a liquid, dissipation the acoustic energy that is associated with it changes the molecular structure of the medium with finite time, which is required for these changes to take place.

The pulse method is much suited to liquids than to gases, mainly because of the much greater absorption of sound by gases, because gases produce much greater damping of the transmitted pulse. The first effect means that the reflector must be of longer duration in order to allow for the greater build-up time of the transducer. These two requirements may be conflicting, because of standing waves that are to be avoided. The distance between transducer and reflector must exceed the length of the transmitted train of waves 
and might thus be such as to give excessive risk of attenuation for satisfactory measurements.

Ultrasonic absorption studies in liquid mixtures can help to determine the behaviour in very fast reaction. In many binary liquid mixtures, the characteristic feature is that there exists a prominent maximum in viscosity, ultra-sonic velocity and sound absorption at an intermediate concentration which was reported by Hemmes and Sarvazyan (1980) and Gerecze (1977), who studied ultrasonic absorption in diethyle amine and water and explained the excess absorption in this system by assuming a simple two-state model involving equilibrium between hydrogen bonds of like and unlike molecules.

In chemical industry, there exists a continuing need for reliable thermodynamic data of binary mixtures. This is particularly true for systems involved in industrial process with their objectives in mind, an attempt has been made to investigate the variations of ultrasonic velocity (v), attenuation coefficient $(\alpha)$, density $(\rho)$ and viscosity $(\eta)$ in aniline and alcohols as a function of concentration and temperature. These systems have a scope for compellation through hydrogen bonding. Ultrasonic absorption studies in liquid mixtures can help in determining the behaviour in very fast reactions. Absorption studies may throw more light on the molecular interaction and hence in this study absorption measurements were taken in aniline with different alcohols at a frequency of $2 \mathrm{MHz}$ and at a temperature of $300.15 \mathrm{~K}$.

The present work has objective to study the behaviour of aniline and alcohols as binary mixtures.

\section{Materials and Methods}

There are two standard techniques for measuring ultrasonic velocity and attenuation. They are:

1. Continuous Wave $(\mathrm{CW})$ method and

2. Pulse-Echo (PE) method.

The $\mathrm{CW}$ method is generally adopted in $\mathrm{kHz}$ regions and in low loss specimens. It is possible to achieve high sensitivity. If sample thickness is too small to provide sufficient separation of pulses in PE method, then the $\mathrm{CW}$ method is better. Except under such special conditions $\mathrm{CW}$ method suffers from:

i) The presence of extra complicating modes of vibrations

ii) Boundary effects iii) The need for larger energy dissipations and subsequent heating of the system

iv) Lack of good accuracy and precision

The PE method, though generally requiring more complex instrumentation, overcome most of the limitations of the $\mathrm{CW}$ method and therefore come into wide spread use.

In the commonly used Pulse-Echo (PE) technique, ultrasonic frequency burst is introduced into the sample through a piezo-electric transducer bonded to the specimen with bonding material. The ultrasonic pulse travels through the sample and an echo is registered each time when it returns to the transducer. More often, accuracy and precision of measurement is limited by the above factors rather than instrument. The errors caused by improper selection of the solid sample, bonding material, transducer etc., are the major items. Ultrasonic technique has been widely used for different types of investigations reported by Kalyansundaram. et al. (1955). In Pulse Echo Overlap method a short sinusoidal electrical wave activates the ultrasonic transducer. The transducer then produces sound wave trains into the liquid inside the cell. This cell is made up of stainless steel which avoids any chemical reactions between the chemicals and the cell.

The ultrasonic velocity was measured with the help of microprocessor based ultrasonic Pulse Echo Overlap (PEO) technique supplied by Roop Telesonic, Bombay at $2 \mathrm{MHz}$ frequency. The internal circuit of PEO is designed with solid state version and it has special memory features of permanent storage and direct digital read out of ultrasonic velocity up to an accuracy of $\pm 1 \mathrm{~m} / \mathrm{s}$.

The PEO method is very versatile and highly accurate technique for measuring the velocity of the ultrasonic waves in materials of liquids and solids structures. A highly absolute accuracy is obtained from this system, since the method is capable of accurately measuring from any cycle of one echo to the corresponding cycle of the next echo. The PEO method is able to handle diffraction (beam spreading) and phase correction properly, so that absolute accuracy of the PEO method may exceed the accuracy of most of the other methods. Reddy and Reddy (1999) improved and used this technique to measure the velocities and attenuations in liquids and solids.

The PEO method may operate either with transducer bonded to the specimen or directly with Radio 
Frequency (RF) bursts. On the negative side, because of transducer effects, the leading cycles of successive echoes are attenuated and trailing cycles are burst up, so that great care has to be taken to obtain proper cycle for cycle match. The phase correction and identification of cycles are to be taken properly which is mentioned in Pulse Superposition technique. In view of the above advantages and accuracy of the system the PEO technique has been adopted for measuring velocity and attenuation.

The binary mixtures of aromatic amine compound (aniline) and alcohols (monohydric) were made into three groups on mixing of aromatic amine compound (Aniline) with aliphatic alcohols (methanol, ethanol and propanol).

The binary liquid was taken into the glass vessel by using $50 \mathrm{~mL}$ pipette and transducer of $2 \mathrm{MHz}$ frequency was immersed into the liquid. The other end of the transducer was connected to the CRO by Bayonet Neill Concelman (BNC) cable and Pulse Echo pattern was observed on the screen. The bottom end of the secondary echo pulse of the wave train was coincided to the gate pulse. The internal circuit of pulse echo overlap system was designed with fully solid state version, which allowed immediate calculation of the ultrasonic wave velocity as given in the following equation.

$$
\mathrm{v}=2 \times l / \mathrm{t}
$$

Where, 1 is the liquid length and $t$ is the time interval.

The densities of all the mixtures were determined with $10 \mathrm{~mL}$ specific gravity bottle and mass $(\mathrm{m})$ of a given volume of the liquid was determined by using a single pan opto-electrical balance. The results of the densities are accurate to $\pm 0.5 \%$.

Viscosity $(\eta)$ measurements were carried out using $20 \mathrm{~mL}$ Cannon-Fenske viscometer. The accuracy of the viscometer for measuring viscosity was $\pm 0.003 \%$ and calibrated by triply distilled water. Cannon -Fenske Viscometer was cleaned with a suitable solvent and evaporated with nitrogen. Liquid whose viscosity is to be determined was introduced into the viscometer through immerse tube 'A' and apply suction 'I', which causes the sample to rise to etched line ' $E$ '. Viscometer was inserted into a holder and it was placed in constant temperature bath. The viscometer was allowed for $10 \mathrm{~min}$ to reach equilibrium at $27^{\circ} \mathrm{C}$ or $15 \mathrm{~min}$ at $98.89{ }^{\circ} \mathrm{C}$. Vertical alignment was accomplished in both by suspending a plumb bob in tube 'I'. In the visco-meter suction was applied to tube and the sample was brought into bulb. The efflux time was measured by allowing the sample into bulb. The effluence time was measured by allowing the sample to flow freely, the time of flow was measured. The experiment was repeated for 7 or 8 times and the average efflux time was recorded as:

Kinematic viscosity $=$ efflux time $\times$ viscometer constant

Dynamic viscosity $(c p)=$ kinematic viscosity $\times$ density

Time of flow of distilled water ( $\mathrm{t}$ ) at $27{ }^{\circ} \mathrm{C}=90$ $\sec$

Instrument constant $(\mathrm{c})=0.0000089$

Kinematic viscosity $=$ efflux time $\times$ viscometer constant $=90 \times 0.0000089=0.008$ poise Dynamic viscosity $(\mathrm{cp})=$ Kinematic viscosity $\times$ density $\left(\mathrm{g} / \mathrm{cm}^{3}\right)$

$($ Expt $)=0.008 \times 1.050=0.008$ poise

Viscosity $(\eta)$ of water at $27^{\circ} \mathrm{C}($ standard $)=0.008$ poise.

The relaxation time $(\tau)$ is calculated using the relation

$$
\tau=4 \eta / 3 d v^{2} \text { sec. }
$$

The long ultrasonic waves were passed through the binary mixture then they were reflected from the reflector face and received by the same transducer, which now acts as a receiver. The transmitted pulse and the received echoes were seen in the CRO screen. Once, the echo pattern achieved on the screen on the CRO, the pattern was adjusted to a suitable height. The transmitted pulse height was measured in $\mathrm{mv} / \mathrm{cm}$ and noted as ' $\mathrm{a}_{0}$ ' on the screen. The height of first echo was noted as ' $a_{1}$ ' and second echo as ' $\mathrm{a}_{2}$ ' and so on. The path length was noted using the micrometer attached to the reflector and is noted as $2 l$.

The attenuation coefficient $(\alpha)$ was calculated as:

$$
\alpha=1 / 2 \ln \log _{e}\left(a_{o} / a_{n}\right) \text { nepers/unit length }
$$

Where, $a_{0} \rightarrow$ amplitude of transmitted pulse

$$
\mathrm{a}_{\mathrm{n}} \rightarrow \text { amplitude of } \mathrm{n}^{\text {th }} \text { echo }
$$

The absorptivity was measured by dividing $\alpha$ by the square of the frequency $(2 \mathrm{MHz})$ of the ultrasonic wave propagated into the liquid media, i.e.

$$
\text { Absorptivity, } \mathrm{A}=\alpha / \mathrm{f}^{2}
$$

Where, $f$ is the frequency of the ultrasonic wave propagated. 


\section{Results and Discussion}

Acoustical and attenuation studies have been carried out for aniline-alcohols mixtures at room temperature of about $300.15 \mathrm{~K}$. The variation of ultrasonic velocity (v) in a solution depends upon the increase or decrease of intermolecular free length $\left(\mathrm{L}_{\mathrm{f}}\right)$ after mixing the components on the basis of a model for sound propagation. Ultrasonic velocity should decrease if the intermolecular free length increases as a result of mixing of components. This in fact observed in the present investigation for all systems which matches with results of Naidu et al. (1990).

Ultrasonic velocities of different mixtures increased with increase in chain length of the alcohols till $40 \%$ of alcohol concentration and beyond $40 \%$ volume component of alcohol there is a reversal trend indicating that the velocities are in the increasing trend of ethanol $>$ propanol $>$ methanol which are reported in Tables 1-3 and the graphs for the ultrasonic velocity is shown in Fig. 1.

In the mixtures of aniline with methanol, ethanol and propanol, the interaction between the $-\mathrm{OH}$ group and the $-\mathrm{NH}_{2}$ group of aniline is maximum. The presence of specific interactions in these two mixtures is explained by the formation of hydrogen bonds between the $\mathrm{H}^{+}$of alcohols and the $\mathrm{N}^{-}$of aniline As the concentration of aniline in the methanol, ethanol and propanol mixtures increases, the filling of voids in the alcohol structure increases the component value of the mixtures. This appears to be ambiguous because the lowest homologue methanol is lowest. But between propanol and ethanol, there is reverse of trend. One of the reasons may be

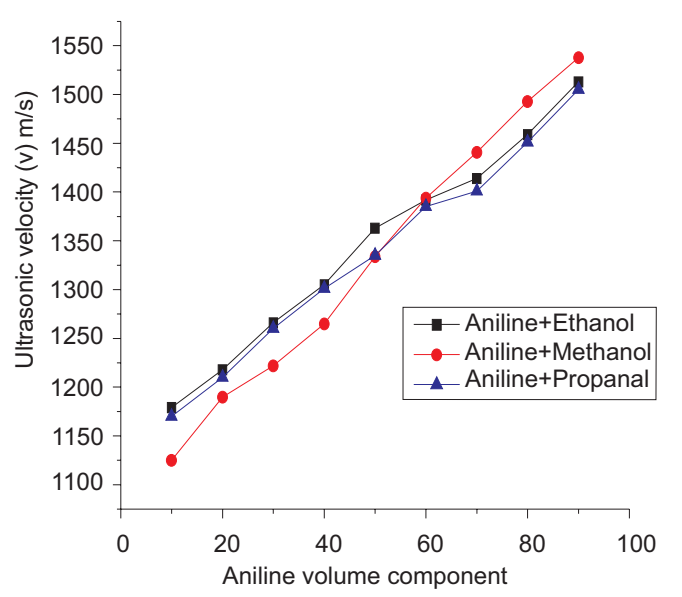

Fig. 1. Aniline volume component vs ultrasonic velocity. beyond $40 \%$ volume component of alcohol the higher homologue propanol is functioning in a coiled manner, apparently behaving much less reactive than ethanol. The trend of velocity variation should have been propanol $>$ ethanol $>$ methanol. Thus straight chain homologue is functioning strongly as far as ultrasonic wave propagation is concerned. These anomalies are also reflected in the measurements. The density values beyond $40 \%$ alcohol are propanol $>$ ethanol $>$ methanol. The graphs for these values are presented Fig. 2. This is in tune with the increase in chain length of the alcohols. These results are reported in the Tables 1-3.

Some of the standardized values of ultrasonic velocity (v) and attenuation coefficient ( $\alpha$ ) of few International standard samples are:

- Ultrasonic velocity (v) of ethyl acetate at $27^{\circ} \mathrm{C}$ (Expt. $)=1103 \mathrm{~m} / \mathrm{s}$

- Ultrasonic velocity (v) of ethyl acetate at $27{ }^{\circ} \mathrm{C}$ (Lit.) $=1108 \mathrm{~m} / \mathrm{s}$

- Attenuation coefficient $(\alpha)$ of ethyl acetate at $27{ }^{\circ} \mathrm{C}($ Expt. $)=0.69 \mathrm{nep} / \mathrm{cm}$

- Attenuation coefficient $(\alpha)$ of ethyl acetate at $27^{\circ} \mathrm{C}$ (Lit.) $=0.70 \mathrm{nep} / \mathrm{cm}$

- Ultrasonic velocity (v) of methyl acetate at $27^{\circ} \mathrm{C}$ (Expt.) $=1120 \mathrm{~m} / \mathrm{s}$

- Ultrasonic velocity (v) of methyl acetate at $27^{\circ} \mathrm{C}$ (Lit.) $=1126 \mathrm{~m} / \mathrm{s}$

- Attenuation coefficient $(\alpha)$ of methyl acetate at $27{ }^{\circ} \mathrm{C}($ Expt. $)=0.68 \mathrm{nep} / \mathrm{cm}$

- Attenuation coefficient $(\alpha)$ of methyl acetate at $27{ }^{\circ} \mathrm{C}$ (Lit.) $=0.67 \mathrm{nep} / \mathrm{cm}$

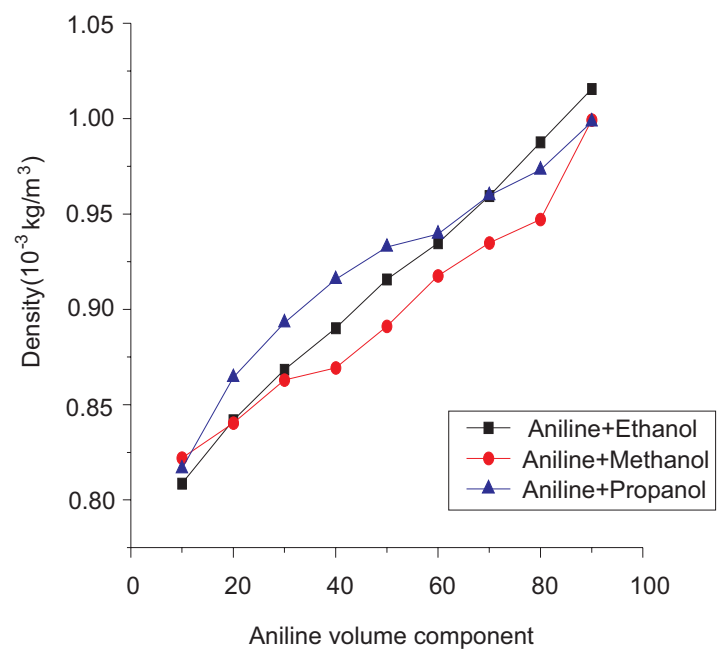

Fig. 2. Aniline volume component vs density. 
Table 1. Ultrasonic velocity, density, viscosity, relaxation time, attenuation coefficient, absorptivity of binay mixture of aniline in methanol at $300.15 \mathrm{~K}$

\begin{tabular}{llllllll}
\hline $\begin{array}{l}\text { Volume component } \\
(\%)\end{array}$ & $\begin{array}{l}\text { Ultrasonic } \\
\text { velocity } \\
(\mathrm{v}) \mathrm{m} / \mathrm{s}\end{array}$ & $\begin{array}{l}\text { Density } \\
(\rho)\end{array}$ & $\begin{array}{l}\text { Viscosity } \\
(\eta)\end{array}$ & $\begin{array}{l}\text { Relaxation } \\
\text { time }(\tau)\end{array}$ & $\begin{array}{l}\text { Attenuation } \\
\text { coefficient } \\
(\alpha) \mathrm{m}^{3}\end{array}$ & $\begin{array}{l}\text { Absorptivity } \\
\text { poise }\end{array}$ & $\begin{array}{l}\text { A } 0^{-8} \mathrm{sec} \\
(\mathrm{A})\end{array}$ \\
\hline 90 & 10 & 1538 & 0.9993 & 0.0248 & 1.398 & 0.5531 & 1.3827 \\
80 & 20 & 1493 & 0.9471 & 0.0204 & 1.2884 & 0.5619 & 1.4304 \\
70 & 30 & 1441 & 0.9348 & 0.0168 & 1.153 & 0.5723 & 1.4307 \\
60 & 40 & 1394 & 0.9176 & 0.0145 & 1.0842 & 0.5716 & 1.429 \\
50 & 50 & 1334 & 0.8911 & 0.0121 & 1.0173 & 0.5860 & 1.465 \\
40 & 60 & 1265 & 0.8693 & 0.0103 & 0.9872 & 0.5991 & 1.4977 \\
30 & 70 & 1222 & 0.8630 & 0.0093 & 0.9622 & 0.6091 & 1.5047 \\
20 & 80 & 1190 & 0.8404 & 0.0082 & 0.9186 & 0.6192 & 1.548 \\
10 & 90 & 1125 & 0.8220 & 0.0072 & 0.9040 & 0.6192 & 1.548 \\
\hline \hline
\end{tabular}

Table 2. Ultrasonic velocity, density, viscosity, relaxation time, attenuation coefficient, absorptivity of binary mixture of aniline in ethanol at $300.15 \mathrm{~K}$

\begin{tabular}{|c|c|c|c|c|c|c|c|}
\hline \multicolumn{2}{|c|}{$\begin{array}{l}\text { Volume component } \\
\qquad(\%)\end{array}$} & \multirow{2}{*}{$\begin{array}{l}\text { Ultrasonic } \\
\text { velocity } \\
\text { (v)m/s }\end{array}$} & \multirow{2}{*}{$\begin{array}{l}\text { Density } \\
(\rho) \\
10^{-3} \mathrm{~kg} / \mathrm{m}^{3}\end{array}$} & \multirow{2}{*}{$\begin{array}{l}\text { Viscosity } \\
(\eta) \\
\text { poise }\end{array}$} & \multirow{2}{*}{$\begin{array}{l}\text { Relaxation } \\
\operatorname{time}(\tau) \\
10^{-8} \mathrm{sec}\end{array}$} & \multirow{2}{*}{$\begin{array}{l}\text { Attenuation } \\
\text { coefficient } \\
(\alpha)\end{array}$} & \multirow{2}{*}{$\begin{array}{l}\text { Absorptivity } \\
\text { (A) } \\
10^{-13}\end{array}$} \\
\hline Aniline & Ethanol & & & & & & \\
\hline 90 & 10 & 1513 & 1.01563 & 0.02968 & 1.7021 & 0.5531 & 1.3827 \\
\hline 80 & 20 & 1459 & 0.98755 & 0.02767 & 1.7550 & 0.5849 & 1.4622 \\
\hline 70 & 30 & 1414 & 0.95947 & 0.02278 & 1.5832 & 0.5849 & 1.4622 \\
\hline 60 & 40 & 1392 & 0.93462 & 0.02185 & 1.6087 & 0.5970 & 1.4925 \\
\hline 50 & 50 & 1363 & 0.91573 & 0.01944 & 1.5236 & 0.6070 & 1.5175 \\
\hline 40 & 60 & 1305 & 0.89014 & 0.01579 & 1.4533 & 0.6192 & 1.548 \\
\hline 30 & 70 & 1266 & 0.86839 & 0.01513 & 1.4495 & 0.6247 & 1.5617 \\
\hline 20 & 80 & 1218 & 0.84193 & 0.01459 & 1.557 & 0.6376 & 1.594 \\
\hline 10 & 90 & 1179 & 0.80863 & 0.01366 & 1.6203 & 0.6378 & 1.594 \\
\hline
\end{tabular}

Table 3. Ultrasonic velocity, density,viscosity, relaxation time, attenuation coefficient, absorptivity of binary mixture of aniline in propanol at $300.15 \mathrm{~K}$

\begin{tabular}{|c|c|c|c|c|c|c|c|}
\hline \multicolumn{2}{|c|}{$\begin{array}{l}\text { Volume component } \\
\qquad(\%)\end{array}$} & \multirow{2}{*}{$\begin{array}{l}\text { Ultrasonic } \\
\text { velocity } \\
\text { (v)m/s }\end{array}$} & \multirow{2}{*}{$\begin{array}{l}\text { Density } \\
(\rho) \\
10^{-3} \\
\mathrm{~kg} / \mathrm{m}^{3}\end{array}$} & \multirow{2}{*}{$\begin{array}{l}\text { Viscosity } \\
(\eta) \\
\text { poise }\end{array}$} & \multirow{2}{*}{$\begin{array}{l}\text { Relaxation } \\
\operatorname{time}(\tau) \\
10^{-8} \mathrm{sec}\end{array}$} & \multirow{2}{*}{$\begin{array}{l}\text { Attenuation } \\
\text { coefficient } \\
(\alpha)\end{array}$} & \multirow{2}{*}{$\begin{array}{l}\text { Absorptivity } \\
\text { (A) } \\
10^{-13}\end{array}$} \\
\hline Aniline & Propanol & & & & & & \\
\hline 90 & 10 & 1505 & 0.9983 & 0.0284 & 1.6746 & 0.5531 & 1.3827 \\
\hline 80 & 20 & 1451 & 0.9731 & 0.0249 & 1.6204 & 0.5970 & 1.4925 \\
\hline 70 & 30 & 1401 & 0.9597 & 0.0204 & 1.4439 & 0.5970 & 1.4925 \\
\hline 60 & 40 & 1385 & 0.9395 & 0.0190 & 1.4057 & 0.6070 & 1.5175 \\
\hline 50 & 50 & 1335 & 0.9327 & 0.0166 & 1.3315 & 0.6192 & 1.5482 \\
\hline 40 & 60 & 1301 & 0.9157 & 0.0144 & 1.2387 & 0.6192 & 1.5482 \\
\hline 30 & 70 & 1260 & 0.8929 & 0.0132 & 1.24156 & 0.6247 & 1.5614 \\
\hline 20 & 80 & 1210 & 0.8644 & 0.0115 & 1.2115 & 0.6376 & 1.5940 \\
\hline 10 & 90 & 1170 & 0.8164 & 0.0111 & 1.19243 & 0.6491 & 1.6227 \\
\hline
\end{tabular}


The attenuation coefficient is a quantity that characterizes how easily a material or medium can be penetrated by a beam of light, sound, particles, or other energy or matter which was reported by Nomoto (1958). A large attenuation coefficient means that the beam is quickly "attenuated" (weakened) as it passes through the medium, and a small attenuation coefficient means that the medium is relatively transparent to the beam. The ultrasonic waves are passed through the specimen under study. They are reflected from the opposite face and received by the same transducer, which now acts as a receiver. The Pulse Echo Wave train pattern is observed on the screen. Gate pulse was made to coincide with the bottom of the secondary echo pulse and amplitude is adjusted to $80 \%$ of its maximum value. The amplitude pulse noted as $\mathrm{a}_{0}$ and the amplitude of the $\mathrm{n}^{\text {th }}$ echo pulse as $a_{n}$. These results were reported by Sathi and Linga (2008; 2007).

The viscosity measurements are reported in Tables 13 and the graphs are shown in Fig. 3.

The relaxation time measurements are reported in Tables 1-3 and the graph is shown in Fig. 4.

A perusal of attenuation coefficient $(\alpha)$ obtained for various binary mixtures of the aniline with alcohols reveals the following factors.

(i) Attenuation coefficients $(\alpha)$ of binary mixture of aniline with methanol is lower while compared to binary mixture of ethanol and propanol with aniline. This means the acidity of alcohol is deciding the attenuation

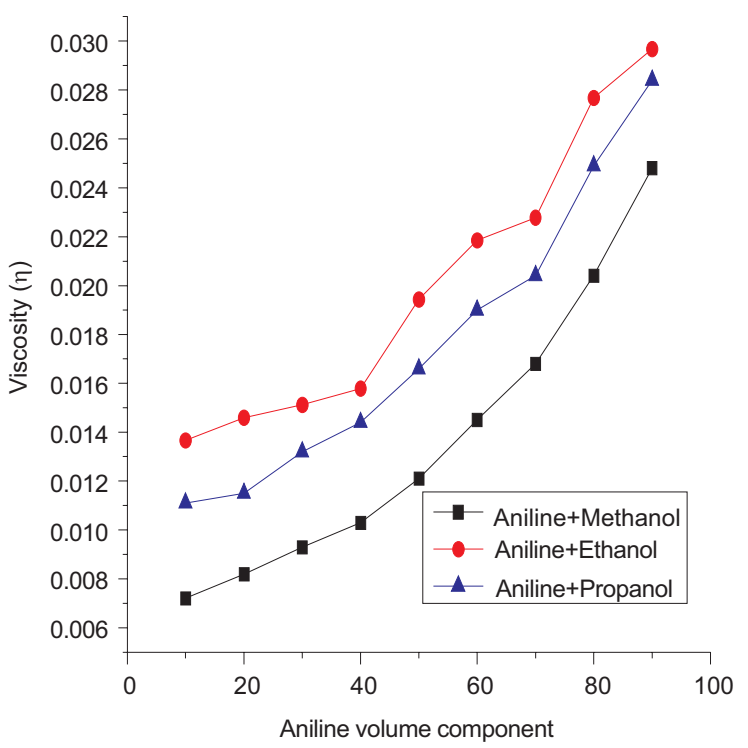

Fig. 3. Aniline volume component vs viscosity. coefficients $(\alpha)$ as observed with ultrasonic velocities (v).

(ii) Attenuation coefficients $(\alpha)$ for propanol is higher than methanol and ethanol as compared

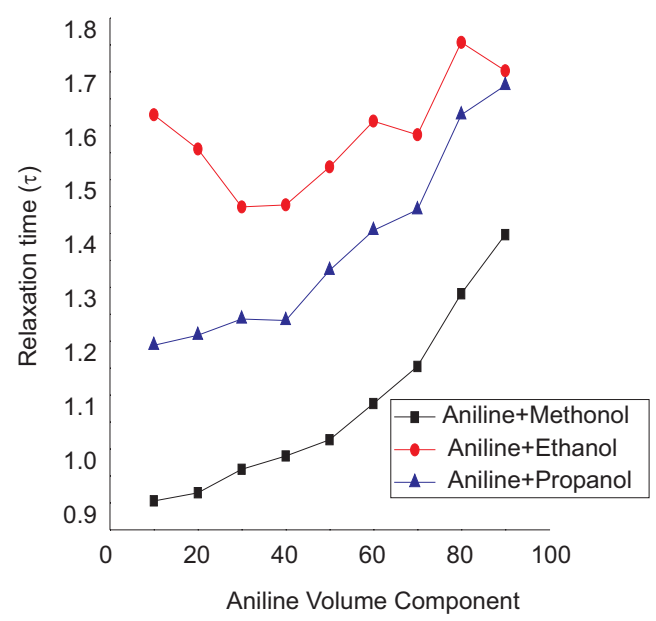

Fig. 4. Aniline volume component vs relaxation time.

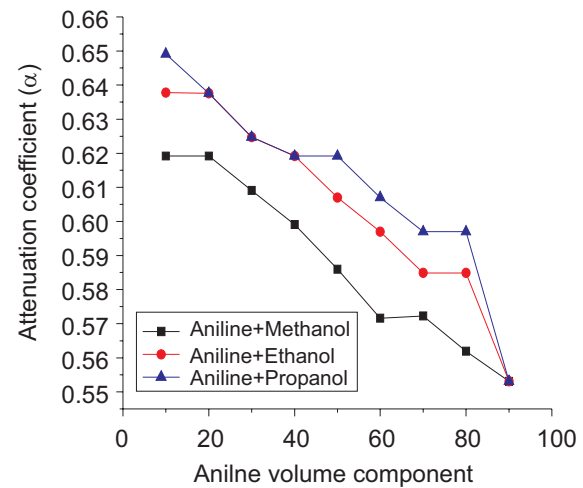

Fig. 5. Aniline volume component vs attenuation coefficient.

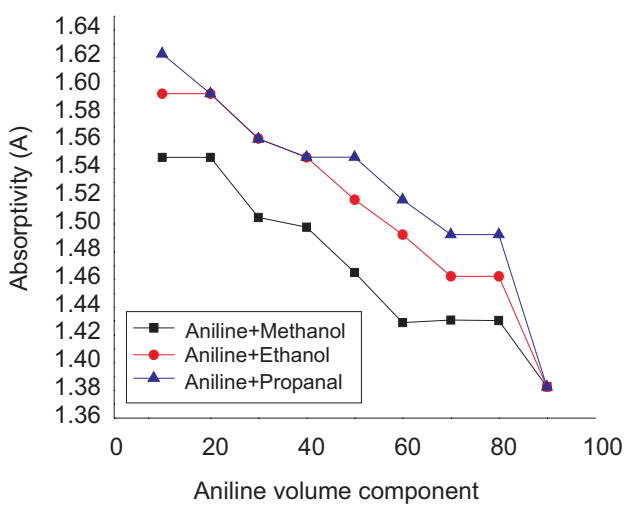

Fig. 6. Aniline volume component vs absorptivity. 
to similar composition of propanol with aniline. The values for the attenuation coefficient and absorptivity are shown in Tables 1-3 and the graphs are shown in Figs. 5-6.

\section{Conclusion}

Thermodynamical and absorption studies are carried out apart from ultrasonic studies on methanol, ethanol and propanol with aniline over entire volume component percentage range. It is found that ultrasonic velocity (v) decreases in aniline-propanol, aniline-ethanol and aniline-methanol systems with increase of concentration of alcohols. It is well known that when alcohols are added to aniline, it effects the properties of the solution either by importing its physical properties to the solution or by causing different interactions among alcohol and aniline species. Attenuation coefficients $(\alpha)$ for propanol is higher than methanol and ethanol. Generally, it can be said that higher the acidity of the alcohol; the higher will be the attenuation coefficient $(\alpha)$. The refractive indices for all the systems are in increasing manner and these values are reported in Tables. In case of methanol, ethanol and propanol, with aniline binary mixtures it is observed that as aniline-propanol $>$ aniline- ethanol $>$ aniline-methanol.

\section{References}

Amis, E.S.J. 1955. Rates mechanisms and solvent. Analytical Chemistry, 27: 1672-1678.

Anantakrishnan, S.V., Anantraman, A.V., 1959. Kientitic studies in ester hydrolysis. Part IX. The hydrolysis of ethyle acetate in Dioxan-water system. Proceedings of the Indian Academy of Sciences, Sec. A, pp. 174-184.

Chavez, M., Habichyan, P., Mass,G.1986. Speed of sound in liquid $\mathrm{CCl}_{3} \mathrm{~F}$ under saturated vapour. Journal of Chemical.Engineering, 31: 291-293.

Durai, S., Ramadas, P. 2004. Optical refractive index from ultrasonic velocity in binary liquid mixtures,
Indian Journal of Pure and Applied Physics, 42: 334-337.

Gerecze, N.G. 1977. Ultrasonic studies in solutions of poly ethyelene glycol, Acoustica, 33: 51-57.

Hemmes, P., Sarvazyan, A.P. 1980. Ultrasonic investigation of solute-solvent interactions in aqueous solution of bases, nucleosides. Journal of Physical Chemistry, 14: 692-696.

Kalyansundaram, S., Manuel Stephen, A., Gopalan, A. 1955. Ultrasonic studies on poly methyl metacrylate in dimethylformamide. Journal of Polymer Matter, 12: 177-181.

Naidu, S.V., Varada R.A.,Chowdoji, R.K. 1990. Ultrasonic velocities and Rao formalism of poly vinylpyrrolidone in N,N-Dimethylformamide solution. Journal of Polymer Matter, 7: 149-153.

Nomoto, O. 1958. Emperical formula for sound velocity in liquid mixtures. Journal of Physical Society, Japan, 13: 1528-1533.

Nomoto, O. 1953. Molecular sound velocity and molecular compressibility of liquid mixtures, Journal of the Physical Society of Japan, 8: 553560.

Reddy, R.B., Reddy, L.D. 1999. Ultrasonic measurements in ethyl acetate and $n$-butanol., Indian Journal of Pure and Applied Physics, 37: 13-19.

Sathi, R.K., Linga, R.D. 2008. Ultrasonic and refractive studies of binary mixtures of aniline in different esters at $300.15 \mathrm{~K}$ temperature. Journal of Acta Ciencia Indica, 3: 435-440.

Sathi, R.K. Linga, R.D. 2007. Acoustical and thermo dynamical studies of binary mixtures of aniline in different esters at $300.15 \mathrm{~K}$ temperature. In: Proceedings of National Symposium on Acoustics (NSA 2007), 511 pp., Acoustical Society of India and New Delhi: Macmillian India.

Tuulmets, A., Salmar, S. 2001. Effect of ultrasound on easter hydrolysis in equeous ethanol. Ultrasonics. Sono-chemistry, 8: 209-212. 